\title{
Electrophysiological insights into connectivity anomalies in schizophrenia: a systematic review
}

\author{
Matteo Maran, Tineke Grent-'t-Jong and Peter J. Uhlhaas*
}

\author{
* Correspondence: \\ peter.uhlhaas@glasgow.ac.uk \\ Institute for Neuroscience and \\ Psychology, Glasgow University, \\ Hillead Str. 58, Glasgow G12 8QB, \\ Scotland
}

\begin{abstract}
The pathophysiology of schizophrenia may fundamentally involve a disturbance in the interaction between neuronal groups that leads to impaired communication within and between brain areas. Such a "dysconnectivity syndrome" could underlie the pronounced cognitive deficits as well as the emergence of psychosis. Evidence for this hypothesis has mainly come from functional magnetic resonance imaging (fMRI) studies which have investigated connectivity anomalies during spontaneous as well as task-related activity. In the current review, we will summarize evidence from electro- and magnetoencephalography (EEG/MEG) which have employed measures, such as coherence, phase-locking and mutual information, that have tested changes in functional connectivity during task-related as well as resting-state time-frequency data. We will highlight the methodological issues associated with these measures as well as provide recommendations for future research.
\end{abstract}

Keywords: Schizophrenia, Dysconnectivity, Functional connectivity, Neural synchrony, EEG, MEG

\section{Background: schizophrenia and neuronal communication}

Schizophrenia (ScZ) is a severe mental disorder characterised by psychotic symptoms and cognitive deficits [1-3]. Cognitive and developmental abnormalities are present in both ScZ-patients [4] and their unaffected siblings [5], suggesting that a combination of shared genetic and environmental risk factors leads to aberrant brain maturation. It is estimated that ScZ affects about $1 \%$ of the population, with a considerable impact on the psychosocial functioning and quality of life [6, 7].

The development of modern structural and functional neuroimaging techniques has highlighted the contribution of aberrant organization and functionality of large-scale networks towards the manifestation of clinical symptoms and cognitive disturbances in ScZ [8]. However, a mechanistic understanding of such anomalies as well as the identification of neuroimaging-biomarkers that could aid early intervention and diagnosis has remained elusive. To address these fundamental questions, it is likely that ScZresearch could benefit from an approach that focuses on the dynamic interactions between multiple regions and neural networks at realistic timescale using advanced Electro/Magnetocencephalography (EEG/MEG). This is because it is now clear that cognition is a complex phenomenon stemming from a spatially and temporally precise

(c) The Author(s). 2016 Open Access This article is distributed under the terms of the Creative Commons Attribution 4.0 International License (http://creativecommons.org/licenses/by/4.0/), which permits unrestricted use, distribution, and reproduction in any medium, provided you give appropriate credit to the original author(s) and the source, provide a link to the Creative Commons license, and indicate if changes were made. The Creative Commons Public Domain Dedication waiver (http://creativecommons.org/ publicdomain/zero/1.0/) applies to the data made available in this article, unless otherwise stated. 
flow of information between and within multiple brain regions [9]. Accordingly, focal anatomical and functional alterations in ScZ may only provide partial insights into the neural origins of the disorder, as the clinical symptoms and cognitive deficits are more likely to stem from a dysconnectivity syndrome whose main feature is an altered communication between brain regions [10-12].

This approach has gained support from recent work which has highlighted that precise temporal correlations are a prerequisite for efficient neuronal communication in large-scale networks $[13,14]$. Emerging evidence suggests that ScZ is associated with alterations in the amplitude and synchrony of rhythms at low- and high-frequencies which could provide a parsimonious mechanisms for the explanation of cognitive deficits and psychosis [15]. Moreover, insights into the rhythm-generating mechanisms underlying neural oscillations and their synchronization across larger areas has identified the contribution of distinct cell-types and transmitter-systems which could establish important links with pre-clinical research that could guide the development of novel treatments [15].

\section{Functional connectivity in EEG/MEG-data: methodological aspects}

While these data provide preliminary support for the notion that ScZ may involve an impairment in neural synchrony, one impediment towards a better understanding of impaired neuronal communication in ScZ is the availability, implementation and interpretation of measures which allow the analysis of connectivity-parameters in EEG/ MEG-data. This issue is crucial since modulations in the amplitude of spectral signals do not per se allow insights into the efficacy of information transmission between neuronal groups. For this to occur, measures of functional interactions need to be implemented. These measures, however, face several methodological challenges.

On a general note, measures of functional connectivity (see Table 1) detect statistical relationships between neural signals over time and thus potentially allow conclusions in

Table 1 EEG/MEG Measures of functional and effective connectivity

\begin{tabular}{|c|c|c|}
\hline Measure & Description & Altered in ScZ \\
\hline Coherence & $\begin{array}{l}\text { A measure of covariation in the amplitude and/or phase } \\
\text { of two neural signals [16] }\end{array}$ & $\bullet \bullet$ \\
\hline Imaginary Part of Coherence & $\begin{array}{l}\text { An estimate of coherence that focuses on time-lagged } \\
\text { synchronization [58] }\end{array}$ & \\
\hline Phase-Locking Value & $\begin{array}{l}\text { A measures of the covariation in the phase of two } \\
\text { neural signals [18] }\end{array}$ & $\bullet \bullet$ \\
\hline Phase-Lag Index & $\begin{array}{l}\text { A measure of the asymmetry of the distribution of } \\
\text { phase differences between two signals [59]. }\end{array}$ & \\
\hline Granger Causality & $\begin{array}{l}\text { Estimates the direction of neural communication } \\
\text { between two time-series through predicting the } \\
\text { course of activation in another cortical area [60] }\end{array}$ & $\bullet$ \\
\hline Transfer Entropy & $\begin{array}{l}\text { A method to estimate directed interactions without a } \\
\text { priori specification of a model for the data [61] }\end{array}$ & \\
\hline Directed Transfer Function & $\begin{array}{l}\text { Comparable method to Granger Causality, but } \\
\text { normalized for inflow from all areas [60] }\end{array}$ & $\bullet$ \\
\hline Mutual Information & $\begin{array}{l}\text { Estimation of amount of signal in first area explained } \\
\text { by the signal in the second area [62] }\end{array}$ & $\bullet$ \\
\hline Synchronization Likelihood & $\begin{array}{l}\text { Normalized strength of mutual information between } \\
\text { signals from two areas [63] }\end{array}$ & $\bullet$ \\
\hline
\end{tabular}

-Preliminary evidence $\bullet \bullet$ Robust Evidence 
regards to the functional interactions between two brain regions. In EEG/MEG-data, the usual starting point for such an approach is first the decomposition of the frequency content of the signal into band-limited spectral values. Traditionally, EEG-data have been examined for changes in coherence between electrodes, where coherences can be defined as the covariation in amplitude and phase between two signals [16]. An important limitation of coherence-measures of scalp EEG/MEG-data are the problem of field spread as sensor-level data represents mixed signals from multiple sources, volume conduction-induced blurring effects, artifact contamination and potential bridging between electrodes in EEG recordings. Moreover, coherence values can be influenced by power fluctuations between two recordings sites, especially if a common source leads to volume conduction across extended areas [17]. Therefore, as an alternative to traditional amplitude-based measures of coherence, more robust measures of phaselocking have been developed, such as the phase-slope index, phase-lag index and imaginary coherence $[18,19]$. While these approaches may provide a more sensitive index for the assessment of synchronization between two recording sites than coherence measures, volume conduction effects may still confound such measures [20, 21].

Importantly, because of their correlational nature, functional connectivity measures do not provide direct information on specific neural interactions and can be affected by spurious relationships between signals, e.g. activity in two regions can be highly correlated even in the absence of a direct communication between them if they are both connected with a third common area [22]. On a more complex level, effective connectivity measures, such as Granger Causality and Directed Transfer Functions, describe the direct interactions between neural assemblies, specifying the direction of information transmission.

The recent development of source connectivity analysis has made it possible to at least partially overcome the traditional problem of field spread/volume conduction and to obtain more precise localization of EEG/MEG-activity [23]. Thus, the adoption of functional connectivity measures, especially at the source level, may have considerable potential for investigating dysconnectivity anomalies in schizophrenia. In the following sections we will review the main findings on functional connectivity abnormalities in the time-frequency domain in the disorder. To this end, we selected studies based on a literature search in PubMed with the following search terms: "schizophrenia", "EEG", "MEG", "oscillations", "synchrony", "long-range synchrony", "functional connectivity", "effective connectivity", "long-range connectivity", "coherence", "phase locking", "phase synchrony", "DCM". Studies with sample sizes $<15$ participants as well as reports that analysed only a narrowly defined frequency bin of $1 \mathrm{~Hz}$ were excluded. Moreover, studies in which EEG/MEG were combined with neuro-stimulation/modulation techniques were not considered.

\section{EEG resting-state connectivity-findings in schizophrenia}

Neural oscillations, especially at alpha-frequencies, are a prominent feature of resting-state activity. Recent MEG-studies of amplitude correlations have revealed resting-state networks similar to those disclosed by Functional Magnetic Resonance Imaging (fMRI) [24, 25].

A large body of work suggests that the resting-state spectral profile in ScZ involves an increase in power at lower (delta $0-4 \mathrm{~Hz}$, and theta $4-7 \mathrm{~Hz}$ ) frequencies [26]. These 
data are complemented by increased connectivity values $[27,28]$ which correlate with decreased cognitive performance [29]. However, the opposite pattern of reduced delta/ theta-band coherence was observed in some studies as well [30-32]. One possibility is that abnormalities in low-frequency connectivity patterns involve regionally specific changes. For example, one study showed that while intra-hemispheric delta-coherence was increased, inter-hemispheric coherence values showed the opposite pattern in ScZ-patients relative to controls [30].

The majority of studies that examined connectivity-measures at alpha-frequencies reported a decrease in ScZ. In particular, decreased alpha-connectivity, as expressed by coherence values as well as lagged coherence and phase synchrony, has been reported at frontal [27, 31], fronto-posterior [27, 28] and parieto-temporal [27] regions (for different findings see [29, 30, 32, 33]). Interestingly, two studies reported a correlation between alpha connectivity at rest and symptom dimension in ScZ [33, 34].

There is conflicting evidence regarding alterations in fast oscillations at beta- (13$30 \mathrm{~Hz}$ ) and gamma- (30-200 Hz) band frequencies at rest, with evidence for both elevated [27], reduced [30] and intact [28, 29, 31, 32] beta-band connectivity. Preliminary evidence suggests that beta-band coherence is influenced by illness progression and clinical symptoms [27]. One study examining the relationship between lagged phase synchrony (LPS) and cortical distance showed a more pronounced decrease of beta 1 $(13-21 \mathrm{~Hz})$ LPS with increasing cortical distance in first-episode psychosis patients (FEP) compared to healthy controls, while opposite findings were obtained for the beta $2(21-30 \mathrm{~Hz})$ band [35]. Similar results in the beta 1 band have been obtained in at-risk subjects who will later develop psychosis [36].

Similar conflicting findings have been reported on measures of gamma-band connectivity, with two studies reporting no difference with controls [30, 32], and one study reporting increases in ScZ-patients with a recent onset and decreases in patients with prolonged illness compared to controls [27]. However, the effect of illness duration is still unclear, since both increases [37] and no differences [28, 29] in gamma-band connectivity measures have been observed in FEP relative to healthy subjects (Table 2).

\section{EEG/MEG parameters during task-related activity in schizophrenia}

Neuronal communication through synchronized, rhythmic activity has been proposed to underlie the emergence of cognitive functions and behaviour [13, 14]. Accordingly, correlations can be expected between alterations in cognitive and perceptual processes

Table 2 Summary of findings on resting-state connectivity in ScZ

\begin{tabular}{|c|c|c|c|c|c|c|c|}
\hline Authors & Technique & Measure & $\delta$ & $\theta$ & a & $\beta$ & $\gamma$ \\
\hline Andreou et al. [29] & EEG & Source-space Imaginary Coherence & $=$ & $\boldsymbol{\Delta}$ & $=$ & $=$ & $=$ \\
\hline Andreou et al. [37] & EEG & Source-space Power-envelope Correlation & - & - & - & - & $\boldsymbol{\Delta}$ \\
\hline Di Lorenzo et al. [27] & EEG & Source-space Lagged Phase Synchrony & $\boldsymbol{\Delta}$ & $\Delta$ & $\nabla$ & $\Delta$ & $\Delta \nabla$ \\
\hline Hinkley et al. [33] & MEG & Global Connectivity & - & - & $\Delta \nabla$ & - & - \\
\hline Kam et al. [30] & EEG & Coherence & $\Delta \nabla$ & $\nabla$ & $\Delta$ & $\nabla$ & $=$ \\
\hline Lehmann et al. [28] & EEG & Source-based Lagged Coherence & $\Delta$ & $=$ & $\nabla$ & $=$ & $=$ \\
\hline Tauscher et al. [31] & EEG & Coherence & $\nabla$ & $\nabla$ & $\nabla$ & $=$ & - \\
\hline Winterer et al. [32] & EEG & Coherence & $\nabla$ & $\nabla$ & $=$ & $=$ & $=$ \\
\hline
\end{tabular}

$\Delta$ Increase, $\boldsymbol{\nabla}$ Decrease, $=$ not altered connectivity, - not reported/investigated 
and changes in EEG/MEG parameters that allow the quantification of interactions between sensor/source-estimates of rhythmic activity.

Several investigations into the possible role of alterations in coherence and synchrony during auditory perception have been conducted, in particular using the Auditory Steady State Paradigm (ASSR). Steady-state responses (SSRs) are evoked oscillatory responses that are entrained to the frequency and phase of temporally modulated stimuli, providing information about the integrity of sensory and perceptual pathways. Alterations in SSRs have been widely documented in ScZ [38], but only few studies investigated connectivity patterns during ASSR. During $40 \mathrm{~Hz}$ ASSRs, decreased phase synchrony between left and right primary auditory cortices has been observed in ScZ [39]. Similarly, decreased fronto-temporal coherence during an auditory oddball task has been observed in ScZ-patients and their siblings [40]. Interestingly, there is preliminary evidence that abnormal interhemispheric alpha coherence during auditory processing may be related to the severity of auditory hallucinations [41], suggesting that aberrant patterns of neuronal communication may also be involved in the positive symptoms of ScZ.

During visual processing, previous studies have focused on the possibility that long-range synchronization during perceptual integration may be impaired at beta/gamma-band frequencies. Two studies [42, 43] reported a reduction of phase-synchronization during visual processing requiring perceptual binding along different stages of the visual hierarchy. Alterations in fronto-posterior high-frequency connectivity in ScZ have been also reported during smooth pursuit eye movements [44], a reliable endophenotype of ScZ. Furthermore, alterations in alpha connectivity have been related to impaired recognition of facial emotional expression in ScZ [45]. Together these findings suggest that abnormal functional interactions as identified by altered coherence and phase-synchronization could be involved in both basic visuo-perceptual deficits and as well contribute to higher cognitive impairments in ScZ.

Finally, there is preliminary evidence on alterations in connectivity changes during higher cognitive functions, such as working memory (WM). Neural synchrony has been proposed as a mechanism for facilitating mnemonic processes [46], and specifically, theta synchrony has been related to retention [47] and central executive processes underlying WM [48]. In line with the dysconnectivity hypothesis, decreased frontoparietal theta phase synchrony was associated with impaired performance in a visual WM task in ScZ-patients [49] (Table 3).

\section{Discussion}

The current overview highlights the possibility of impaired functional connectivity at different frequencies in ScZ. Given the potential importance of aberrant neuronal communication towards the emergence of cognitive deficits and clinical symptoms [10-12], we believe it is important to pursue this approach further albeit considering several methodological and conceptual caveats for the analysis and interpretation of functional connectivity measures in EEG/MEG-data.

This is particularly the case for resting-state measurements which currently provide conflicting evidence for the presence and direction of effects in functional connectivity parameters of rhythmic activity in ScZ. Similarly to coherence and phase-synchrony estimates during task-related oscillations, volume conduction effects can lead to spurious 
Table 3 Summary of findings on task-related connectivity in ScZ

\begin{tabular}{|c|c|c|c|c|}
\hline Function & Authors & Technique & Measure & Increase/decrease \\
\hline Auditory Processing & Henshall et al. [41] & EEG & Coherence & $\boldsymbol{\nabla} a$ \\
\hline Auditory Processing & Mulert et al. [39] & EEG & $\begin{array}{l}\text { Source-space Lagged } \\
\text { Phase synchrony }\end{array}$ & $\boldsymbol{\nabla} \gamma$ \\
\hline Auditory Processing & Winterer et al. [40] & EEG & Coherence & $\boldsymbol{\nabla} \delta$ \\
\hline Face Processing & Lee et al. [42] & EEG & $\begin{array}{l}\text { Event-Related Phase } \\
\text { Coherence }\end{array}$ & $\boldsymbol{\nabla} \gamma$ \\
\hline Face Processing & Uhlhaas et al. [43] & EEG & Phase Synchrony & $\boldsymbol{\nabla} \beta$ \\
\hline $\begin{array}{l}\text { Smooth Pursuit Eye } \\
\text { Movement }\end{array}$ & Krishna et al. [44] & EEG & Phase Synchrony & $\boldsymbol{\nabla} \beta \gamma$ \\
\hline Facial Emotional Processing & Popov et al. [45] & MEG & $\begin{array}{l}\text { Graph-based Index of Global } \\
\text { Connectivity }\end{array}$ & $\Delta \boldsymbol{\nabla} a$ \\
\hline Working Memory & Griesmayr et al. [49] & EEG & Phase-Locking Value & $\boldsymbol{\nabla} \ominus$ \\
\hline
\end{tabular}

$\Delta$ Increase, $\boldsymbol{\nabla}$ Decrease, $=$ intact connectivity

correlations between EEG/MEG-sensor estimates for resting-state measurements [17] This may be particularly the case in scenarios where the spectral content of timefrequency data is already different between clinical and non-clinical populations and thus lead to biased estimates of connectivity parameters that do not reflect "true" alterations in the interaction between neuronal channels but rather the impact of widely different spectra.

One solution to this problem is the transformation of EEG/MEG-data into sourcespace which allows the reconstruction of the underlying generators which can then be further interrogated for connectivity parameters. Difficulties with this approach are the limited spatial resolution which can impede the separation of sources in nearby brain regions as well as similar contributions of volume conduction towards phase-estimates, for example, of time-series data. Recent approaches towards this problem have seen the removal of signals with zero-phase lag through orthogonalization [25] as well as the investigation of power-correlations of EEG/MEG source-reconstructed signals [50]. However, such approaches are still yet to be applied to ScZ research (see [51-53] for findings in Dementia and Epilepsy).

In addition, future research should also carefully consider the analysis of connectivity parameters of high-frequency activity at rest. Recent findings have highlighted the importance of careful artefact removal from EEG/MEG-recordings to diminish the influence of cranial and muscle activity towards estimates of beta/gamma-band activity [54]. Although MEG-data is faced with similar problems as in EEG-recordings [55], there is evidence to suggest that EEG may be characterized by reduced signal-to-noise for estimating gamma-band fluctuations [56]. This is a particular pertinent issue in measuring high-frequency activity during spontaneous activity because of the small contribution of beta/gamma-band fluctuations towards the measured EEG/MEG-signal which makes the separation of gamma-band activity due to neural vs. non-neural activity particularly challenging. While this problem has been mainly addressed in the context of time-frequency analysis of EEG/MEG-data, we would like to note that it is likely that the contamination of electrophysiological signals through cranial and muscle artefacts can also lead to spurious correlations between EEG/MEG signals. There is emerging evidence that the contribution of such artefacts towards resting-state connectivity estimates at frequencies $>30 \mathrm{~Hz}$ may be higher in EEG-data [50]. 
While the current findings of task-related rhythmic activity in ScZ are slightly more consistent than data from resting-state activity in highlighting a reduced functional coupling between brain areas, we would like to highlight that similar methodological issues also apply to the analysis of connectivity parameters during cognitive and perceptual paradigms which, in our view, have been insufficiently addressed. One advantage of analysis of task-related rhythmic activity is that stimulus-induced oscillations lead to higher changes in the spectral content of EEG/MEG-data than ongoing-spontaneous oscillations which facilitates the separation of artefacts from signals due to neural processes, especially if baseline or pre-stimulus activity is subtracted from post-stimulus activity.

A potential caveat for the analysis of connectivity-parameters during task-related EEG/MEG-data is, however, that stimuli that are associated with large transient activity as is the case during visual and auditory paradigms, can lead to spurious correlations between sensor/source-signals. This is less the case where task-related activity predominantly involves intrinsic oscillations which occur during higher cognitive processes, such as attention, memory and executive processes [57]. To improve the ability to identify "true" neuronal interactions from EEG/MEG-data, analysis should focus on frequencies that show distinct peaks in the time-frequency content of the signal that point towards taskrelated modulation of ongoing rhythmic activity. Further investigation of such distinct peaks with connectivity estimates may then allow physiologically meaningful insights into the structure of neuronal communication and its disturbances in ScZ. Given the difficulties of identifying such patterns from EEG/MEG sensor-data, we believe that future studies should preferentially employ such analysis at the source-level.

\section{Conclusions}

We believe that a focus on identifying aberrant patterns of neuronal communication in EEG/MEG-data has considerable potential for ScZ-research. The current evidence suggests that such abnormalities are present in functional connectivity indexes both during restingstate and task-related activity across different frequencies with considerable inconsistencies in terms of the direction of effects. As a result, for these approaches to be useful measures for early intervention and development of biomarkers, the methodological issues raised need to be addressed as well as standardized protocols should be implemented to facilitate replicability across samples.

\footnotetext{
Abbreviations

ASSR: Auditory steady state paradigm; DCM: Dynamic causal modelling; EEG: Electroencephalography; FEP: First episode psychosis; fMRI: Functional magnetic resonance imaging; Hz: Hertz; LPS: Lagged phase synchrony; MEG: Magnetoencephalography; ScZ: Schizophrenia; SSR: Steady-state response; UHR: Ultra-high risk for psychosis; WM: Working memory

Acknowledgements

N/A.

Authors' contributions

All authors contributed equally to this work. All authors read and approved the final manuscript.

Competing interests

The authors declare that they have no competing interests.

Received: 8 August 2016 Accepted: 19 October 2016

Published online: 05 November 2016

References

1. Insel TR. Rethinking schizophrenia. Nature. 2010;468(7321):187-93.
} 
2. Keefe RS, Harvey PD. Cognitive impairment in schizophrenia. Handb Exp Pharmacol. 2012;213:11-37. doi:10.1007/978-3-642-25758-2_2

3. Picchioni MM, Murray RM. Schizophrenia. BMJ. 2007;335(7610):91-5.

4. Reichenberg A, Caspi A, Harrington H, Houts R, Keefe RS, Murray RM, et al. Static and dynamic cognitive deficits in childhood preceding adult schizophrenia: a 30-year study. Am J Psychiatry. 2010;167(2):160-9.

5. Barch DM, Cohen R, Csernansky J. Altered cognitive development in the siblings of individuals with schizophrenia. Clin Psychol Sci. 2014;2(2):138-51.

6. Kuperberg G, Heckers S. Schizophrenia and cognitive function. Curr Opin Neurobiol. 2000;10(2):205-10.

7. Ventura J, Hellemann GS, Thames AD, Koellner V, Nuechterlein KH. Symptoms as mediators of the relationship between neurocognition and functional outcome in schizophrenia: a meta-analysis. Schizophr Res. 2009:113(2-3):189-99.

8. Fornito A, Zalesky A, Pantelis C, Bullmore ET. Schizophrenia, neuroimaging and connectomics. Neuroimage. 2012; 62(4):2296-314.

9. Varela F, Lachaux JP, Rodriguez E, Martinerie J. The brainweb: phase synchronization and large-scale integration. Nat Rev Neurosci. 2001;2(4):229-39.

10. Friston KJ. Schizophrenia and the disconnection hypothesis. Acta Psychiatr Scand Suppl. 1999;395:68-79.

11. Friston KJ, Frith CD. Schizophrenia: a disconnection syndrome? Clin Neurosci. 1995;3(2):89-97.

12. Stephan KE, Friston KJ, Frith CD. Dysconnection in schizophrenia: from abnormal synaptic plasticity to failures of self-monitoring. Schizophr Bull. 2009;35(3):509-27.

13. Fries P. A mechanism for cognitive dynamics: neuronal communication through neuronal coherence. Trends Cogn Sci. 2005;9(10):474-80.

14. Fries P. Rhythms for cognition: communication through coherence. Neuron. 2015:88(1):220-35.

15. Uhlhaas PJ, Singer W. Abnormal neural oscillations and synchrony in schizophrenia. Nat Rev Neurosci. 2010;11(2):100-13.

16. Bowyer SM. Coherence a measure of the brain networks: past and present. Neuropsychiatr Electrophysiol. 2016; 2(1). doi: 10.1186/s40810-015-0015-7.

17. Winter WR, Nunez PL, Ding J, Srinivasan R. Comparison of the effect of volume conduction on EEG coherence with the effect of field spread on MEG coherence. Statist Med. 2007;26:3946-57.

18. Lachaux JP, Rodriguez E, Martinerie J, Varela FJ. Measuring phase synchrony in brain signals. Hum Brain Mapp. 1999:8(4):194-208

19. van Diessen E, Numan T, van Dellen E, van der Kooi AW, Boersma M, Hofman D, et al. Opportunities and methodological challenges in EEG and MEG resting state functional brain network research. Clin Neurophys. 2015;126:1468-81.

20. Guevara R, Velazquez JL, Nenadovic V, Wennberg R, Senjanovic G, Dominguez LG. Phase synchronization measurements using electroencephalographic recordings: what can we really say about neuronal synchrony? Neuroinformatics. 2005;3(4):301-14.

21. Nunez PL, Srinivasan R, Westdorp AF, Wijesinghe RS, Tucker DM, Silberstein RB, et al. EEG coherency. I: Statistics, reference electrode, volume conduction, Laplacians, cortical imaging, and interpretation at multiple scales. Electroencephalogr Clin Neurophysiol. 1997;103(5):499-515.

22. Friston KJ. Functional and effective connectivity: a review. Brain Connect. 2011;1(1):13-36.

23. Schoffelen JM, Gross J. Source connectivity analysis with MEG and EEG. Hum Brain Mapp. 2009;30(6):1857-65.

24. Brookes MJ, Woolrich M, Luckhoo H, Price D, Hale JR, Stephenson MC, et al. Investigating the electrophysiological basis of resting state networks using magnetoencephalography. Proc Natl Acad Sci U S A. 2011;108(40):16783-8.

25. Hipp JF, Hawellek DJ, Corbetta M, Siegel M, Engel AK. Large-scale cortical correlation structure of spontaneous oscillatory activity. Nat Neurosci. 2012:15(6):884-90.

26. Boutros NN, Arfken C, Galderisi S, Warrick J, Pratt G, lacono W. The status of spectral EEG abnormality as a diagnostic test for schizophrenia. Schiz Res. 2008;99(1-3):225-37.

27. Di Lorenzo G, Daverio A, Ferrentino F, Santarnecchi E, Ciabattini F, Monaco L, et al. Altered resting-state EEG source functional connectivity in schizophrenia: the effect of illness duration. Front Hum Neurosci. 2015;9:234.

28. Lehmann D, Faber PL, Pascual-Marqui RD, Milz P, Herrmann WM, Koukkou M, et al. Functionally aberrant electrophysiological cortical connectivities in first episode medication-naive schizophrenics from three psychiatry centers. Front Hum Neurosci. 2014:8:635.

29. Andreou C, Leicht G, Nolte G, Polomac N, Moritz S, Karow A, et al. Resting-state theta-band connectivity and verbal memory in schizophrenia and in the high-risk state. Schizophr Res. 2015;161(2-3):299-307.

30. Kam JW, Bolbecker AR, O'Donnell BF, Hetrick WP, Brenner CA. Resting state EEG power and coherence abnormalities in bipolar disorder and schizophrenia. J Psychiatr Res. 2013:47(12):1893-901.

31. Tauscher J, Fischer P, Neumeister A, Rappelsberger P, Kasper S. Low frontal electroencephalographic coherence in neuroleptic-free schizophrenic patients. Biol Psychiatry. 1998;44(6):438-47.

32. Winterer G, Egan MF, Radler T, Hyde T, Coppola R, Weinberger DR. An association between reduced interhemispheric EEG coherence in the temporal lobe and genetic risk for schizophrenia. Schizophr Res. 2001;49(1-2):129-43.

33. Hinkley LB, Vinogradov S, Guggisberg AG, Fisher M, Findlay AM, Nagarajan SS. Clinical symptoms and alpha band resting-state functional connectivity imaging in patients with schizophrenia: implications for novel approaches to treatment. Biol Psychiatry. 2011;70(12):1134-42.

34. Merrin EL, Floyd TC. Negative symptoms and EEG alpha in schizophrenia: a replication. Schizophr Res. 1996; 19(2-3):151-61.

35. Ramyead A, Studerus E, Kometer M, Heitz U, Gschwandtner U, Fuhr P, et al. Neural oscillations in antipsychoticnaive patients with a first psychotic episode. World J Biol Psychiatry. 2016;17(4):296-307.

36. Ramyead A, Kometer M, Studerus E, Koranyi S, Ittig S, Gschwandtner U, et al. Aberrant current source-density and lagged phase synchronization of neural oscillations as markers for emerging psychosis. Schizophr Bull. 2015;41(4):919-29.

37. Andreou C, Nolte G, Leicht G, Polomac N, Hanganu-Opatz IL, Lambert M, et al. Increased resting-state gamma-band connectivity in first-episode schizophrenia. Schizophr Bull. 2015;41(4):930-9.

38. Brenner CA, Krishnan GP, Vohs JL, Ahn WY, Hetrick WP, Morzorati SL, et al. Steady state responses: electrophysiological assessment of sensory function in schizophrenia. Schizophr Bull. 2009;35:1065-77. 
39. Mulert C, Kirsch V, Pascual-Marqui R, McCarley RW, Spencer KM. Long-range synchrony of gamma oscillations and auditory hallucination symptoms in schizophrenia. Int J Psychophysiol. 2011;79(1):55-63.

40. Winterer G, Coppola R, Egan MF, Goldberg TE, Weinberger DR. Functional and effective frontotemporal connectivity and genetic risk for schizophrenia. Biol Psychiatry. 2003;54(11):1181-92.

41. Henshall KR, Sergejew AA, Rance G, McKay CM, Copolov DL. Interhemispheric EEG coherence is reduced in auditory cortical regions in schizophrenia patients with auditory hallucinations. Int J Psychophysiol. 2013;89(1):63-71.

42. Lee SH, Kim DW, Kim EY, Kim S, Im CH. Dysfunctional gamma-band activity during face structural processing in schizophrenia patients. Schizophr Res. 2010;119(1-3):191-7.

43. Uhlhaas PJ, Linden DE, Singer W, Haenschel C, Lindner M, Maurer K, et al. Dysfunctional long-range coordination of neural activity during Gestalt perception in schizophrenia. J Neurosci. 2006;26(31):8168-75.

44. Krishna N, O’Neill H, Sanchez-Morla EM, Thaker GK. Long range frontal/posterior phase synchronization during remembered pursuit task is impaired in schizophrenia. Schizophr Res. 2014;157(1-3):198-203.

45. Popov TG, Rockstroh BS, Popova P, Carolus AM, Miller GA. Dynamics of alpha oscillations elucidate facial affect recognition in schizophrenia. Cogn Affect Behav Neurosci. 2014;14(1):364-77.

46. Fell J, Axmacher N. The role of phase synchronization in memory processes. Nat Rev Neurosci. 2011;12(2):105-18.

47. Sarnthein J, Petsche H, Rappelsberger P, Shaw GL, von Stein A. Synchronization between prefrontal and posterior association cortex during human working memory. Proc Natl Acad Sci U S A. 1998;95(12):7092-6.

48. Sauseng P, Klimesch W, Schabus M, Doppelmayr M. Fronto-parietal EEG coherence in theta and upper alpha reflect central executive functions of working memory. Int J Psychophysiol. 2005;57(2):97-103.

49. Griesmayr B, Berger B, Stelzig-Schoeler R, Aichhorn W, Bergmann J, Sauseng P. EEG theta phase coupling during executive control of visual working memory investigated in individuals with schizophrenia and in healthy controls. Cogn Affect Behav Neurosci. 2014;14(4):1340-55.

50. Siems M, Pape AA, Hipp JF, Siegel M. Measuring the cortical correlation structure of spontaneous oscillatory activity with EEG and MEG. Neurolmage. 2016;129:345-55.

51. Cuesta P, Garcés P, Castellanos NP, López ME, Aurtenetxe S, Bajo R, et al. Influence of the APOE $\varepsilon 4$ allele and mild cognitive impairment diagnosis in the disruption of the MEG resting state functional connectivity in sources space. J Alzheimers Dis. 2015;44(2):493-505.

52. Elisevich K, Shukla N, Moran JE, Smith B, Schultz L, Mason K, et al. An assessment of MEG coherence imaging in the study of temporal lobe epilepsy. Epilepsia. 2011;52(6):1110-9.

53. Englot DJ, Rolston JD, Wang DD, Kirsch HE, Nagarajan SS, Chang EF. 206 spikes, slowing, and functional connectivity: multimodal magnetoencephalography in epilepsy surgery. Neurosurgery. 2016;63 Suppl 1:181.

54. Hipp JF, Siegel M. Dissociating neuronal gamma-band activity from cranial and ocular muscle activity in EEG. Front Hum Neurosci. 2013;7:338.

55. Carl C, Acik A, Konig P, Engel AK, Hipp JF. The saccadic spike artifact in MEG. Neuroimage. 2012;59(2):1657-67.

56. Muthukumaraswamy SD, Singh KD. Visual gamma oscillations: the effects of stimulus type, visual field coverage and stimulus motion on MEG and EEG recordings. Neuroimage. 2013;69:223-30.

57. Tallon-Baudry C, Bertrand O. Oscillatory gamma activity in humans and its role in object representation. Trends Cogn Sci. 1999;3(4):151-62.

58. Nolte G, Bai O, Wheaton L, Mari Z, Vorbach S, Hallett M. Identifying true brain interaction from EEG data using the imaginary part of coherency. Clin Neurophysiol. 2004;115(10):2292-307.

59. Stam CJ, Nolte G, Daffertshofer A. Phase lag index: assessment of functional connectivity from multi channel EEG and MEG with diminished bias from common sources. Hum Brain Mapp. 2007;28(11):1178-93.

60. Blinowska KJ. Review of the methods of determination of directed connectivity from multichannel data. Med Biol Eng Comput. 2011;49(5):521-9.

61. Vicente R, Wibral M, Lindner M, Pipa G. Transfer entropy - a model-free measure of effective connectivity for the neurosciences. J Comput Neurosci. 2011;30:45-67.

62. Fraser AM, Swinney HL. Independent coordinates for strange attractors from mutual information. Phys Rev A Gen Phys. 1986:33:1134-40

63. Stam CJ, Van Dijk BW. Synchronization likelihood: an unbiased measure of generalized synchronization in multivariate data sets. Physica D. 2002;163:236-51.

\section{Submit your next manuscript to BioMed Central and we will help you at every step:}

- We accept pre-submission inquiries

- Our selector tool helps you to find the most relevant journal

- We provide round the clock customer support

- Convenient online submission

- Thorough peer review

- Inclusion in PubMed and all major indexing services

- Maximum visibility for your research

Submit your manuscript at www.biomedcentral.com/submit 